\title{
Cryptococcal meningitis in HIV (AIDS) patients: A Mini Review
}

\author{
Manodeep Sen*, Tanushri Chatterji, Vivek Singh, Pushpa Yadav, Anupam Das and Vineeta Mittal \\ Department of Microbiology, Dr. Ram Manohar Lohia Institute of Medical Sciences (RMLIMS), Lucknow, India
}

\begin{abstract}
Meningitis is a major health problem causing inflammation of the protective membranes covering the brain and spinal cord, collectively known as the meninges. Meningitis is generally caused by Meningococcus, Streptococcus pyogenes bacterial species causing bacterial meningitis: BM), Mycobacterium tuberculosis (Tubercular meningitis: TBM), Cryptococcus neoformans (fungal meningitis: CM). In recent times the CM and TBM are two most common types of chronic infectious meningitis. In adults, HIV-associated Cryptococcal meningitis has been reported in many areas of the world having high HIV seroprevalence i.e. in Sub-Saharan Africa. Cryptococcal meningitis is the fourth most commonly recognized cause of life-threatening infection among AIDS patients.Despite availability of antiretroviral therapy, the rise in rate of infections due to HIV/AIDS, suppress the cell mediated immune system and subjects are predisposed for opportunistic infections. Additionally, In AIDS patients, it is characterized by lack of meningeal signs and diminished inflammatory response. Diagnosis of CM is generally based on the collection of CSF sample and/or blood samples. Initially the CSF samples are processed for 'gold standard' culture methods, microscopy and India ink preparation. Serologically, Latex agglutination test (LAT) is the most commonly used for detection of Cryptococcal capsular antigen. Enzyme immunoassay (EIA) is another serological tool for detection of capsular polysaccharide antigens of C. neoformans in CSF.
\end{abstract}

Keywords: Cryptococcal Meningitis, HIV-Associated

\section{Introduction}

Meningitis is a major health problem causing inflammation of the protective membranes covering the brain and spinal cord, collectively known as the meninges[1] Meningitis is generally caused by Meningococcus, Streptococcus pyogenes bacterial species causing bacterial meningitis: BM), Mycobacterium tuberculosis (Tubercular meningitis: TBM), Cryptococcus neoformans (fungal meningitis: $\mathrm{CM})$. In recent times the CM and TBM are two most common types of chronic infectious meningitis[2]. In adults, HIV-associated cryptococcal meningitis has been reported in many areas of the world having high HIV seroprevalence i.e. in Sub-Saharan Africa[3, 4]. Though once known to be rare, cryptococcosis has occurred at a high frequency in India in the past two decades[5, 6].Furthermore, prevalence of HIV-associated CM is also found to be common in many parts of India including North-eastern and as well as in Southern India [7, 8].Cryptococcal meningitis is the fourth most commonly recognized cause of life-threatening infection among AIDS patients[9].Despite availability of antiretroviral therapy, the rise in rate of infections due to HIV/AIDS, suppress the cell mediated immune system and subjects are predisposed for opportunistic infections. Additionally, In AIDS patients, it is characterized by lack of meningeal signs and diminished inflammatory response[10].

Clinical picture compatible with a diagnosis of Cryptococcal meningitis includes evidence of fever/ headache/meningismus/altered mental status or any other neurological manifestation with either a cerebrospinal fluid (CSF) abnormal biochemistry and/or pleocytosisand with a cryptococcal antigen titer of $\geq 1: 8$ and/or Positive India Ink preparation for capsulated yeast cells and/or a Positive CSF culture yielding Cryptococcus neoformans[11].

Diagnosis of $\mathrm{CM}$ is generally based on the collection of CSF sample and/or blood samples. Initially the CSF samples are processed for 'gold standard' culture methods, microscopy and India ink preparation. Serologically, Latex agglutination test (LAT) is the most commonly used for detection of Cryptococcal capsular antigen[12]. Enzyme immunoassay (EIA) is another serological tool for detection of capsular polysaccharide antigens of $C$. neoformans in CSF. This is a rapid test that provides visual and numeric result in less than an hour without pre-treatment of the specimen[13, 14].

\section{Conclusion}

Since there is limited literature available on Cryptococcal meningitis and its causative agent (Cryptococcus neoformans) therefore, further studies focusing on the significance based on diagnosis, pathogenesis related to the infection would be highly appreciated.

\section{Acknowledgements}

The authors acknowledge full support of Department of Microbiology, Dr. Ram Manohar Lohia Institute of Medical Sciences (RMLIMS), Vibhuti Khand, Gomti Nagar. Lucknow. 


\section{Reference}

1. Sáez-Llorens X, McCracken GH. Bacterial meningitis in children.Lancet2003; 361:2139-2148.

2. Qu J, Zhou T, Zhong C, Deng R, Lü X. Comparison of clinical features and prognostic factors in HIV-negative adults with cryptococcal meningitis and tuberculous meningitis: a retrospective study.BMC infectious disease2017; 17:51.

3. Williamson PR, Jarvis JN, Panackal AA, et al. Cryptococcal meningitis: epidemiology, immunology, diagnosis and therapy.Nature reviews Neurology2017; 13:13-24.

4. Britz E, Perovic O, von Mollendorf $\mathrm{C}$, et al. The Epidemiology of Meningitis among Adults in a South African Province with a High HIV Prevalence, 2009-2012. PloS one2016; 11:e0163036.

5. Banerjee U, Gupta K, Chatterjee B, Sethi S. Cryptococcosis at AIIMS.The National medical journal of India1994; 7:51-52.

6. Banerjee U, Datta K, Majumdar T, Gupta K. Cryptococcosis in India: the awakening of a giant?Medical mycology2001; 39:51-67.

7. Sharma SR, Hussain M, Habung H. Neurological manifestations of HIV-AIDS at a tertiary care institute in North Eastern India.Neurology India2017; 65:64-68.
8. Indira P, Kumar PM, Shalini S, Vaman K. Opportunistic Infections among People Living with HIV (PLHIV) with Diabetes Mellitus (DM) Attending a Tertiary Care Hospital in Coastal City of South India.PloS one2015; 10:e136280.

9. Kwon-Drung KJ, Bennette JE, editors, Medical mycology. London: Lea and Febiger, 1992.

10. Chuck SL, Sande MA. Infections with Cryptococcus neoformans in the acquired immunodeficiency syndrome The New England journal of medicine1989; 32:794-799.

11. Coen M, O'Sullivan M, Bubb WA, Kuchel PW, Sorrell T. Proton nuclear magnetic resonance-based metabonomics for rapid diagnosis of meningitis and ventriculitis.Clinical infectious disease2005; 41:1582-1590.

12. Feldmesser M, Harris C, Reichberg S, Khan S, Casadevall A. Serum Cryptococcal Antigen in Patients with AIDS. Clinical infectious disease1996; 23:827-830.

13. Casadevall A, Mukherjee J, Scharff MD. Monoclonal antibody based ELISAs for cryptococcal polysaccharide. Journal of immunological methods 1992; 154:27-35.

14. Sekhon AS, Garg AK, Kaufman L, Kobayashi GS, Hamir Z JM, Moledina N.Evaluation of a commercial enzyme immunoassay for the detection of cryptococcal antigen. Mycoses 1993; 36:31-34.

*Corresponding author:

Dr. Manodeep Sen, Professor (junior grade), Department of Microbiology, Dr. Ram Manohar Lohia Institute of Medical Sciences (RMLIMS), Vibhuti Khand, Gomti Nagar, Lucknow- 226010 (INDIA),

Phone: +919839446858

Email: sen_manodeep6@yahoo.com 